\title{
Higher frequency of chromosomal aberrations in ovarian endometriosis compared to extragonadal endometriosis: a possible link to endometrioid adenocarcinoma
}

\author{
Meike Körner ${ }^{1}$, Elisabeth Burckhardt ${ }^{1}$ and Luca Mazzucchelli ${ }^{2}$ \\ ${ }^{1}$ Institute of Pathology, University of Bern, Bern, Switzerland and ${ }^{2}$ Istituto Cantonale di Patologia, Locarno, \\ Switzerland
}

\begin{abstract}
Endometriosis may progress to invasive endometrioid adenocarcinoma, particularly in the ovary. Up to now, little is known of the molecular mechanisms possibly involved in the malignant transformation of endometriosis. Therefore, in this study, extragonadal endometriosis $(n=10)$, ovarian endometriosis without malignancy $(n=10)$, ovarian endometriosis with direct transition into endometrioid adenocarcinoma $(n=8)$, and normal endometrium $(n=12)$ were investigated for numerical chromosomal aberrations by fluorescence in situ hybridization using centromere enumeration probes. The proportions of cells with aneusomies were semiquantitatively assessed. Trisomies 1 and 7 , and monosomies 9 and 17 were found in endometriosis, ovarian endometrioid adenocarcinoma, and normal endometrium. The proportions of aneusomic cells were significantly higher in ovarian endometrioid carcinoma compared with ovarian endometriosis $(P<0.001)$, and in ovarian endometriosis compared with extragonadal endometriosis and normal endometrium $(\boldsymbol{P}<0.001)$. The data provide new evidence of a common lineage of endometriosis and ovarian endometrioid carcinoma. The higher frequency of chromosomal aberrations in endometrioid carcinoma than in endometriosis may reflect an expansion of aberrant cell clones already present in endometriosis during the progression to cancer. The higher frequency of chromosomal aberrations in ovarian endometriosis than in extragonadal endometriosis suggests a role of the ovarian stromal milieu in the induction of genetic changes, which may eventually lead to invasive cancer.
\end{abstract}

Modern Pathology (2006) 19, 1615-1623. doi:10.1038/modpathol.3800699; published online 15 September 2006

Keywords: endometriosis; endometriod adenocarcinoma; ovary; FISH

Endometriosis is associated with endometrioid adenocarcinoma, particularly in the ovary and more rarely at extragonadal sites. ${ }^{1,2}$ Several epidemiological and morphological observations suggest that endometriosis is indeed a precursor of ovarian endometrioid carcinoma. Endometriosis and ovarian carcinoma share many risk factors, ${ }^{2}$ and endometriosis is significantly more common in patients with ovarian endometrioid carcinoma than in patients with ovarian serous or mucinous carcinoma. ${ }^{3}$ Furthermore, in early stage ovarian endometrioid carcinoma, a direct transition from benign endometriosis to carcinoma can be observed microscopically. ${ }^{4}$ However, so far there is only little data linking endometriosis and cancer at the molecular

Correspondence: Dr M Körner, MD, Institute of Pathology, University of Bern, Murtenstrasse 31, 3010 Bern, Switzerland. E-mail: meike.koerner@pathology.unibe.ch

Received 6 June 2006; revised 8 July 2006; accepted 21 August 2006; published online 15 September 2006 level. For instance, one study showed that synchronous endometriosis and carcinoma share many loss of heterozygosity (LOH) events when present in the same ovary, but not when occurring in contralateral ovaries. $^{5}$

Recently, multiple numerical chromosomal aberrations were found in benign ovarian structures, namely in the ovarian surface epithelium and in cortical inclusion cysts. ${ }^{6}$ The same aneusomies were also found, at a higher frequency, in ovarian serous tumors, providing a link at the molecular level between the ovarian surface epithelium and cortical inclusion cysts on the one hand and ovarian serous cancer on the other. In addition, aneusomies were found to be more frequent in inclusion cysts than in the surface epithelium, suggesting a role of the specialized ovarian stromal milieu, to which inclusion cysts are more exposed than the surface epithelium, in the development of genomic changes. ${ }^{7}$ In analogy, it can be hypothesized that endometriosis as a putative precursor of ovarian 
cancer may harbor chromosomal aberrations associated with malignancy. ${ }^{8,9}$ Indeed, several chromosomal aberrations, in particular monosomy 17, have been described in endometriosis. ${ }^{10-12}$ However, whether these aberrations are associated with malignant transformation remains unknown. Furthermore, it is unclear whether chromosomal aberrations in endometriosis occur mainly in the ovary, or if they can also be found in endometriosis outside the ovary.

The aims of the present study were: first, to analyze a subset of numerical chromosomal aberrations in endometriosis; second, to investigate whether the identified chromosomal aberrations provide evidence of a link between endometriosis and ovarian endometrioid adenocarcinoma; and, finally, to look for differences, with respect to chromosomal aberrations, between ovarian endometriosis and extragonadal endometriosis that might result from putative interactions with the specialized ovarian environment. As to technical approach, we opted for fluorescence in situ hybridization (FISH) since this method allows a good correlation of the results with morphology and offers several advantages in the evaluation of genomic changes in small precursor lesions over other techniques, such as conventional cytogenetic analysis, comparative genomic hybridization, or LOH analysis. ${ }^{6,13}$

\section{Materials and methods}

\section{Tissue Samples}

Formalin-fixed, paraffin-embedded tissue samples were obtained from surgical resection specimens. The following cases were analyzed: extragonadal endometriosis in the peritoneum, bladder wall, appendix, colon, and abdominal wall $(n=10)$; ovarian endometriosis occurring in the absence of any malignancy $(n=10)$; ovarian endometriotic cysts in direct continuity with primary ovarian endometrioid adenocarcinoma, meeting Sampson's and Scott's criteria for carcinoma arising in endometriosis $^{14,15}(n=8)$; and normal endometrium from women in the reproductive phase and in the postmenopause $(n=12)$. Of note, in the investigated endometriosis samples, neither hyperplasia nor cytological atypia was present.

The study conformed to the ethical guidelines of the Institute of Pathology, University of Bern, Switzerland, and of the Istituto Cantonale di Patologia, Locarno, Switzerland, and was reviewed by the respective Institutional Review Boards.

\section{FISH Studies}

Based on haematoxylin and eosin (H\&E)-stainings, we selected representative tissue sections of endometriosis, endometrioid carcinoma, and normal endometrium. Adjacent serial tissue sections were hybridized with combinations of two differentially labeled centromere probes for chromosomes 1, 7, 9, and 17 (Vysis Inc., Downer's Grove, IL, USA). The centromere probes were selected according to numerical aberrations found in ovarian endometrioid carcinoma in previous studies ${ }^{16-19}$ and in own preliminary investigations. Standard chromosome preparations of peripheral blood lymphocytes were used as controls for probe specificities in each hybridization procedure. The procedure was carried out as described previously ${ }^{20}$ and indicated by the manufacturer. Four-micrometer-thick tissue sections were dewaxed, pretreated, and then denatured at $73^{\circ} \mathrm{C}$ in a $70 \%$ formamide $/ 2 \times$ SSC solution for $5 \mathrm{~min}$. After dehydration and treatment with proteinase $\mathrm{K}$, the tissue was exposed to the hybridization mixture overnight at $37^{\circ} \mathrm{C}$. The slides were then washed and counterstained with DAPI II $(125 \mathrm{ng} / \mathrm{ml}$; Vysis Inc.) in an antifade solution. The signals were evaluated by one experienced investigator (EB) according to established criteria. ${ }^{21}$ To avoid misinterpretations, only cells with at least one bright signal were evaluated. Two signals were counted as one if they lay very close $(\leq 0.5 \mu \mathrm{m})$ to each other. In each case, at least 100 cells in endometriosis and/or carcinoma or endometrium were counted, and the percentages of nuclei with one, two, or three signals were recorded. Endometrial epithelial cells and endometrial stromal cells were evaluated separately in endometriosis and in the endometrium.

In each case, normal control tissues were evaluated. These included cortical stromal cells in the ovary, connective tissue cells adjacent to extraovarian endometriosis, and myometrial smooth muscle cells adjacent to the endometrium. For all probes, the average numbers of nuclei with one, two, and three signals did not differ significantly between these control tissues, providing evidence of the consistency of the procedures. The control tissues served as reference for the determination of the significance of chromosomal gains and losses in the investigated tissues.

\section{Statistics}

A statistical analysis was performed to evaluate whether the percentages of nuclei with one or three signals differed significantly between control tissues, extragonadal endometriosis, ovarian endometriosis, ovarian carcinoma, and normal endometrium. For this purpose, the Student's $t$-test was used; $P<0.05$ was considered to be statistically significant. To confirm the results of the Student's $t$ test, it was also calculated whether the differences in the mean percentages of nuclei with one or three signals between the investigated groups exceeded two standard deviations from the mean; this was always the case when the Student's $t$-test yielded $P<0.05$. 


\section{Results}

Trisomies 1 and 7 and monosomies 9 and 17 were found in extragonadal endometriosis, ovarian endometriosis, ovarian endometrioid adenocarcinoma, and normal endometrium: these tissues harbored significantly more nuclei with three signals for chromosomes 1 and 7 and one signal for chromosomes 9 and 17 in epithelial cells (Table 1) and stromal cells (Table 2) than the control tissues (Table 3) $(P<0.001)$.

The chromosomal aberrations in the epithelial cells are shown in Figure 1, exemplified by an endometriotic cyst (left column) and an endometrioid adenocarcinoma (right column). Figure 2 shows the semiquantitative analysis of FISH signals found in the epithelial cells. The proportions of epithelial cells with chromosomal aberrations varied significantly among the different tissues. All aneusomies occurred in a significantly larger percentage of cells in ovarian endometriosis than in the normal endometrium $(P<0.001$ for each aneusomy). Furthermore, all chromosomal aberrations were also found in larger proportions of cells in ovarian endometriosis than in extragonadal endometriosis $(P<0.001$ for each aneusomy), whereas no clear differences were observed between extragonadal endometriosis and the normal endometrium. Moreover, the frequencies of chromosomal aberrations increased in a significant manner from ovarian endometriosis to ovarian endometrioid adenocarcinoma $(P<0.001$ for each aneusomy). Of note, there were no significant differences between ovarian endometriosis associated with carcinoma and ovarian endometriosis without carcinoma with respect to the proportions of cells with trisomies 1 and 7 and monosomies 9 and 17; therefore, ovarian endometriosis with and without carcinoma was considered as one group in the statistical analyses.

In endometriosis and normal endometrium, the endometrial stromal cells showed the same chromosomal aberrations as the epithelial cells as well as similar differences in the proportions of aneusomic cells between ovarian endometriosis, extragonadal endometriosis, and normal endometrium. This is illustrated in Figure 3. The proportions of aneusomic stromal cells were significantly higher in ovarian endometriosis than in extragonadal endometriosis and normal endometrium $(P<0.001$ for each aneusomy). Conversely, the differences between extragonadal endometriosis and normal endometrium were of no significance. Furthermore, the comparison of Figures 2 and 3 shows that the fractions of aneusomic cells were lower in the endometrial stroma were in the corresponding epithelium.

We considered the possibility that interpretative issues could affect the results obtained on tissues with a low rate of aneusomic cells, such as normal endometrium and extragonadal endometriosis. In order to exclude artifacts due to nucleus truncation in tissue sections or over-interpretation of split signals, ${ }^{21}$ we compared the fractions of cells with chromosomal aberrations in endometriosis and the normal endometrium with those found in adjacent normal tissues, such as ovarian stromal cells, connective tissue cells, and myometrial smooth muscle cells. In the latter, one or three centromere signals occurred in no more than $6 \%$ of cells, that is, in significantly smaller fractions of cells than in the epithelium and stroma of endometriosis and normal endometrium $(P<0.001$ for each chromosome; Figures 2 and 3 ).

Interestingly, not all normal tissues were disomic. While the ovarian stroma, connective tissue, and smooth muscle did not appear to harbor significant numbers of aneusomic cells, the normal endometrium, in comparison, showed substantial fractions of cells with trisomies 1 and 7 , and monosomies 9 and $17(P<0.001)$. The occurrence of chromosomal aberrations in the endometrium did not depend on the reproductive phase; there were no differences in the amount of aneusomic cells between the endometrium of women of reproductive age and the atrophic endometrium of post-menopausal women.

\section{Discussion}

This study shows the presence of different chromosomal aberration rates in endometriosis at extragonadal sites, endometriosis in the ovary, ovarian endometrioid adenocarcinoma associated with endometriosis, and in the normal endometrium. The results allow significant insights into the pathology of endometriosis. First, chromosomal aberrations, namely trisomies 1 and 7 and monosomies 9 and 17 , which are frequent in ovarian endometrioid adenocarcinoma also occur at lower rates in endometriosis. This provides evidence at the molecular level that endometriosis may be a precursor of ovarian endometrioid adenocarcinoma and that a chromosomal instability in endometriosis may favor malignant transformation. Second, the semiquantitative analysis of FISH results demonstrates a higher frequency of chromosomal aberrations in ovarian endometriosis compared to extragonadal endometriosis. This suggests a role of the special ovarian milieu in the induction of genetic changes. Finally, the study shows that a semiquantitative analysis of FISH results allows detection of chromosomal aberrations in small lesions and in normal tissues like the endometrium that cannot be routinely investigated with other molecular techniques.

The present findings are consistent with and add to previous investigations linking ovarian cancer and endometriosis at the molecular level. In fact, endometriosis and endometrioid carcinoma were previously shown to share many LOH events involving the same alleles, when located in the same ovary, whereas no common $\mathrm{LOH}$ events were found when endometriosis was present in one ovary 
Table 1 Fluorescent signals in epithelial cells of extraovarian endometriosis, ovarian endometriosis not associated with carcinoma, ovarian endometriosis associated with carcinoma, ovarian endometrioid adenocarcinoma, and normal endometrium (\% of nuclei with 1 , 2 , and 3 signals)

\begin{tabular}{|c|c|c|c|c|c|c|c|c|c|c|c|c|}
\hline \multirow[t]{2}{*}{ Case } & \multicolumn{12}{|c|}{ Number of signals } \\
\hline & 1 & $\begin{array}{l}2 \\
\text { Chromosome } 1\end{array}$ & 3 & 1 & $\begin{array}{l}2 \\
\text { Chromosome } 7\end{array}$ & 3 & 1 & $\begin{array}{c}2 \\
\text { Chromosome } 9\end{array}$ & 3 & 1 & $\begin{array}{c}2 \\
\text { Chromosome } 17\end{array}$ & 3 \\
\hline \multicolumn{13}{|c|}{ Extraovarian endometriosis } \\
\hline 1 & 5 & 47 & 21 & 4 & 76 & 20 & 39 & 61 & 0 & 40 & 60 & 0 \\
\hline 2 & 5 & 79 & 16 & 2 & 82 & 16 & 34 & 65 & 1 & 32 & 68 & 0 \\
\hline 3 & 3 & 82 & 15 & 5 & 79 & 16 & 33 & 67 & 0 & 30 & 70 & 0 \\
\hline 4 & 5 & 78 & 17 & 6 & 75 & 19 & 37 & 63 & 0 & 31 & 68 & 1 \\
\hline 5 & 4 & 77 & 19 & 5 & 77 & 18 & 33 & 66 & 1 & 33 & 65 & 2 \\
\hline 6 & 4 & 80 & 16 & 4 & 79 & 17 & 37 & 61 & 2 & 34 & 65 & 1 \\
\hline 7 & 5 & 77 & 18 & 4 & 77 & 19 & 34 & 65 & 1 & 35 & 65 & 0 \\
\hline 8 & 4 & 77 & 19 & 4 & 77 & 19 & 36 & 63 & 1 & 33 & 66 & 1 \\
\hline 9 & 3 & 72 & 25 & 3 & 70 & 27 & 54 & 46 & 0 & 52 & 47 & 1 \\
\hline 10 & 5 & 75 & 20 & 2 & 77 & 21 & 41 & 59 & 0 & 40 & 60 & 0 \\
\hline
\end{tabular}

Ovarian endometriosis not associated with carcinoma

$\begin{array}{llllll}11 & 3 & 72 & 25 & 3 & 70 \\ 12 & 4 & 69 & 27 & 4 & 70 \\ 13 & 5 & 67 & 28 & 4 & 67 \\ 14 & 3 & 68 & 29 & 6 & 76 \\ 15 & 4 & 71 & 25 & 3 & 69 \\ 16 & 2 & 72 & 26 & 3 & 73 \\ 17 & 2 & 71 & 27 & 4 & 72 \\ 18 & 4 & 67 & 29 & 3 & 69 \\ 19 & 3 & 70 & 27 & 3 & 73 \\ 20 & 3 & 60 & 37 & 5 & 57\end{array}$

$\begin{array}{llllllll}70 & 27 & 54 & 46 & 0 & 46 & 53 & 1 \\ 70 & 26 & 57 & 43 & 0 & 48 & 51 & 1 \\ 67 & 29 & 50 & 48 & 2 & 48 & 52 & 0 \\ 76 & 18 & 51 & 48 & 1 & 45 & 55 & 0 \\ 69 & 28 & 51 & 49 & 0 & 45 & 53 & 2 \\ 73 & 24 & 52 & 48 & 0 & 52 & 47 & 1 \\ 72 & 24 & 58 & 42 & 0 & 56 & 44 & 0 \\ 69 & 28 & 60 & 40 & 0 & 56 & 44 & 0 \\ 73 & 24 & 57 & 43 & 0 & 59 & 40 & 1 \\ 57 & 38 & 55 & 45 & 0 & 52 & 48 & 0\end{array}$

Ovarian endometriosis associated with carcinoma

$\begin{array}{lllll}21 & 3 & 69 & 28 & 3 \\ 22 & 4 & 71 & 25 & 3 \\ 23 & 5 & 66 & 29 & 5 \\ 24 & 4 & 96 & 27 & 2 \\ 25 & 3 & 75 & 27 & 4 \\ 26 & 3 & 68 & 29 & 5 \\ 27 & 3 & 70 & 27 & 3 \\ 28 & 3 & 72 & 25 & 3\end{array}$

$\begin{array}{ll}68 & 29 \\ 70 & 27 \\ 71 & 24 \\ 68 & 30 \\ 75 & 21 \\ 68 & 27 \\ 69 & 28 \\ 73 & 24\end{array}$

29
27
24
30
21
27
28
24

$\begin{array}{ll}56 & 43 \\ 55 & 45 \\ 52 & 48 \\ 43 & 17 \\ 52 & 48 \\ 53 & 46 \\ 51 & 49 \\ 52 & 48\end{array}$

$\begin{array}{ll}1 & 45 \\ 0 & 45 \\ 0 & 43 \\ 0 & 44 \\ 0 & 53 \\ 1 & 53 \\ 0 & 50 \\ 0 & 52\end{array}$

$\begin{array}{ll}55 & 0 \\ 55 & 0 \\ 57 & 0 \\ 56 & 0 \\ 47 & 0 \\ 47 & 0 \\ 50 & 0 \\ 47 & 1\end{array}$

Ovarian endometrioid adenocarcinoma

$\begin{array}{llllll}21 & 4 & 57 & 39 & 3 & 61 \\ 22 & 3 & 61 & 36 & 3 & 61 \\ 23 & 3 & 56 & 41 & 3 & 62 \\ 24 & 2 & 85 & 40 & 3 & 63 \\ 25 & 2 & 61 & 37 & 4 & 62 \\ 26 & 3 & 57 & 40 & 5 & 57 \\ 27 & 3 & 57 & 40 & 3 & 54 \\ 28 & 2 & 63 & 35 & 4 & 62\end{array}$

$\begin{array}{ll}61 & 36 \\ 61 & 36 \\ 62 & 35 \\ 63 & 34 \\ 62 & 34 \\ 57 & 38 \\ 54 & 43 \\ 62 & 34\end{array}$

$\begin{array}{ll}65 & 35 \\ 69 & 31 \\ 70 & 30 \\ 68 & 32 \\ 65 & 35 \\ 65 & 35 \\ 65 & 35 \\ 66 & 34\end{array}$

$\begin{array}{ll}0 & 52 \\ 0 & 58 \\ 0 & 58 \\ 0 & 53 \\ 0 & 63 \\ 0 & 64 \\ 0 & 66 \\ 0 & 72\end{array}$

$\begin{array}{ll}47 & 1 \\ 42 & 0 \\ 42 & 0 \\ 47 & 0 \\ 37 & 0 \\ 36 & 0 \\ 33 & 1 \\ 28 & 0\end{array}$

Normal endometrium

\begin{tabular}{|c|c|c|c|c|c|c|c|c|c|c|c|c|}
\hline 29 & 4 & 78 & 18 & 6 & 74 & 20 & 37 & 62 & 1 & 38 & 62 & 0 \\
\hline 30 & 4 & 76 & 20 & 4 & 77 & 19 & 32 & 68 & 0 & 31 & 69 & 0 \\
\hline 31 & 5 & 77 & 18 & 4 & 77 & 19 & 31 & 68 & 1 & 30 & 70 & 0 \\
\hline 32 & 5 & 73 & 22 & 4 & 73 & 23 & 35 & 63 & 2 & 38 & 62 & 0 \\
\hline 33 & 6 & 74 & 20 & 3 & 88 & 19 & 33 & 67 & 0 & 34 & 66 & 0 \\
\hline 34 & 5 & 77 & 18 & 5 & 76 & 19 & 30 & 69 & 1 & 31 & 69 & 0 \\
\hline 35 & 4 & 73 & 23 & 4 & 72 & 24 & 33 & 66 & 1 & 34 & 66 & 0 \\
\hline 36 & 4 & 72 & 24 & 5 & 72 & 23 & 34 & 65 & 1 & 37 & 63 & 0 \\
\hline 37 & 4 & 78 & 18 & 3 & 78 & 19 & 28 & 72 & 0 & 29 & 71 & 0 \\
\hline 38 & 4 & 75 & 21 & 4 & 75 & 21 & 30 & 96 & 1 & 29 & 71 & 0 \\
\hline 39 & 5 & 77 & 18 & 5 & 75 & 20 & 26 & 74 & 0 & 28 & 72 & 0 \\
\hline 40 & 4 & 74 & 22 & 5 & 74 & 21 & 32 & 68 & 0 & 34 & 66 & 0 \\
\hline
\end{tabular}

and the carcinoma in the contralateral one. ${ }^{5,22}$ Furthermore, the same $\mathrm{X}$ chromosome allele was inactivated in endometriosis and carcinoma occurring in the same ovary, whereas different $\mathrm{X}$ chromo- some alleles were inactivated when endometriosis was present in one ovary and the carcinoma in the other one. ${ }^{5}$ We detected higher frequencies of the same genomic aberrations in ovarian endometrioid 
Table 2 Fluorescent signals in stromal cells of extraovarian endometriosis, ovarian endometriosis not associated with carcinoma, ovarian endometriosis associated with carcinoma, and normal endometrium (\% of nuclei with 1, 2 , and 3 signals)

Case

Number of signals

\begin{tabular}{cccccccccc}
\hline 1 & 2 & 3 & 1 & 2 & 3 & 1 & 2 & 3 & 1 \\
Chromosome 1 & & & Chromosome 7 & & & Chromosome 9 & & & Chromosome 17 \\
\hline
\end{tabular}

\begin{tabular}{l}
\hline Extraovarion \\
1 \\
2 \\
3 \\
4 \\
5 \\
6 \\
7 \\
8 \\
9 \\
10
\end{tabular}

ian endometriosis

$\begin{array}{ll}5 & 83 \\ 4 & 85 \\ 4 & 85 \\ 5 & 84 \\ 5 & 83 \\ 4 & 85 \\ 5 & 83 \\ 5 & 84 \\ 5 & 78 \\ 4 & 84\end{array}$

$\begin{array}{ll}83 & 11 \\ 85 & 11 \\ 85 & 11 \\ 84 & 11 \\ 83 & 12 \\ 85 & 11 \\ 83 & 12 \\ 84 & 11 \\ 78 & 17 \\ 84 & 12\end{array}$

11
11
11
11
12
11
12
11
17
12

4
3
4
4
4
3
3
5
3
5

84
85
85
84
83
87
86
84
85
81

$\begin{array}{ll}12 & 27 \\ 12 & 19 \\ 11 & 18 \\ 12 & 20 \\ 13 & 17 \\ 10 & 21 \\ 11 & 20 \\ 11 & 21 \\ 12 & 19 \\ 14 & 28\end{array}$

73
81
82
80
83
79
79
79
81
72

Ovarian endometriosis not associated with carcinoma

11
12
13
14
15
16
17
18
19
20

$\begin{array}{lll}5 & 78 & 17 \\ 4 & 80 & 16 \\ 5 & 77 & 18 \\ 4 & 80 & 16 \\ 6 & 76 & 18 \\ 4 & 79 & 17 \\ 5 & 77 & 18 \\ & \text { NA } & \\ 4 & 78 & 18 \\ 5 & 70 & 25\end{array}$

$\begin{array}{lc}3 & 81 \\ 4 & 81 \\ 4 & 77 \\ 6 & 76 \\ 5 & 78 \\ 3 & 82 \\ 4 & 81 \\ & \text { NA } \\ 6 & 77 \\ 5 & 69\end{array}$

$\begin{array}{ll}16 & 36 \\ 15 & 42 \\ 19 & 34 \\ 18 & 33 \\ 17 & 36 \\ 15 & 39 \\ 15 & 42 \\ 17 & 35 \\ 26 & 39\end{array}$

64
57
66
66
64
61
58
NA
65
61

Ovarian endometriosis associated with carcinoma

$\begin{array}{lll}21 & 5 & 79 \\ 22 & 5 & 79 \\ 23 & 6 & 77 \\ 24 & 5 & 76 \\ 25 & 5 & 80 \\ 26 & 6 & 77 \\ 27 & 4 & 78 \\ 28 & & \text { NA }\end{array}$

$\begin{array}{ll}79 & 16 \\ 79 & 16 \\ 77 & 17 \\ 76 & 19 \\ 80 & 15 \\ 77 & 17 \\ 78 & 18 \\ \text { NA } & \end{array}$

$\begin{array}{ll}6 & 77 \\ 5 & 78 \\ 5 & 80 \\ 6 & 77 \\ 5 & 77 \\ 6 & 76 \\ 6 & 76 \\ & \text { NA }\end{array}$

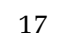

17
17
15

17
18

18

18
18

Normal endometrium

\begin{tabular}{|c|c|c|c|c|c|c|c|c|c|c|c|c|}
\hline 29 & 5 & 38 & 12 & 5 & 82 & 13 & 25 & 75 & 0 & 27 & 73 & 0 \\
\hline 30 & 5 & 82 & 13 & 5 & 83 & 12 & 22 & 78 & 0 & 23 & 77 & 0 \\
\hline 31 & 5 & 83 & 12 & 5 & 83 & 12 & 20 & 79 & 1 & 20 & 80 & 0 \\
\hline 32 & 5 & 82 & 13 & 5 & 81 & 14 & 25 & 75 & 0 & 27 & 73 & 0 \\
\hline 33 & 4 & 87 & 14 & 4 & 83 & 13 & 24 & 76 & 0 & 25 & 75 & 0 \\
\hline 34 & 6 & 82 & 12 & 4 & 83 & 13 & 20 & 79 & 1 & 22 & 78 & 0 \\
\hline 35 & 3 & 82 & 15 & 4 & 83 & 13 & 21 & 79 & 0 & 23 & 77 & 0 \\
\hline 36 & 4 & 82 & 14 & 5 & 81 & 14 & 20 & 80 & 0 & 22 & 78 & 0 \\
\hline 37 & 5 & 82 & 13 & 4 & 83 & 13 & 14 & 85 & 1 & 17 & 83 & 0 \\
\hline 38 & 5 & 83 & 12 & 4 & 84 & 12 & 18 & 82 & 0 & 20 & 80 & 0 \\
\hline 39 & 5 & 82 & 13 & 5 & 83 & 12 & 15 & 85 & 0 & 17 & 83 & 0 \\
\hline 40 & 5 & 83 & 12 & 3 & 86 & 11 & 19 & 81 & 0 & 20 & 80 & 0 \\
\hline
\end{tabular}

$\mathrm{NA}=$ not assessable because of small number of nuclei.

carcinoma than in ovarian endometriosis, which suggests an expansion during carcinogenesis of aberrant cell clones already present in endometriosis. Similarly, previous studies showed that the LOH events present in both ovarian endometriosis and an associated endometrioid carcinoma occurred in higher incidence in the carcinoma. ${ }^{5}$

There is increasing evidence from the present study and from other cytogenetic investigations that multiple numerical chromosomal aberrations occur in endometriosis. We found that trisomies and monosomies are present in all investigated endometriosis cases. Similarly, monosomy 17 was previously reported to occur with a $100 \%$ incidence in endometriosis. ${ }^{11,12}$ In another study, trisomy 11 and monosomy 16 were found in addition to monosomy 17 in endometriosis. ${ }^{10}$ However, in the latter study, chromosomal aberrations occurred at lower incidences and in smaller proportions of cells compared with the present results. The different chromosomal aberration rates between studies may be due to differences in the hybridization efficacies 
Table 3 Fluorescent signals in control cells adjacent to extraovarian endometriosis, ovarian endometriosis, and normal endometrium (\% of nuclei with 1, 2, and 3 signals)

\begin{tabular}{|c|c|c|c|c|c|c|c|c|c|c|c|c|}
\hline \multirow[t]{2}{*}{ Case } & \multicolumn{12}{|c|}{ Number of signals } \\
\hline & 1 & $\begin{array}{c}2 \\
\text { Chromosome } 1\end{array}$ & 3 & 1 & $\begin{array}{l}2 \\
\text { Chromosome } 7\end{array}$ & 3 & 1 & $\begin{array}{c}2 \\
\text { Chromosome } 9\end{array}$ & 3 & 1 & $\begin{array}{c}2 \\
\text { Chromosome } 17\end{array}$ & 3 \\
\hline \multicolumn{13}{|c|}{ Connective tissues adjacent to extraovarian endometriosis } \\
\hline 1 & 4 & 96 & 0 & 4 & 96 & 0 & 5 & 95 & 0 & 4 & 96 & 0 \\
\hline 2 & 5 & 95 & 0 & 4 & 96 & 0 & 5 & 95 & 0 & 7 & 93 & 0 \\
\hline 3 & 5 & 95 & 0 & 4 & 85 & 11 & 4 & 96 & 0 & 3 & 97 & 0 \\
\hline 4 & 6 & 94 & 0 & 5 & 95 & 0 & 3 & 97 & 0 & 4 & 96 & 0 \\
\hline 5 & 4 & 96 & 0 & 5 & 95 & 0 & 4 & 95 & 1 & 3 & 97 & 0 \\
\hline 6 & 5 & 95 & 0 & 5 & 95 & 0 & 3 & 97 & 0 & 4 & 96 & 0 \\
\hline 7 & 5 & 95 & 0 & 4 & 96 & 0 & 3 & 97 & 0 & 4 & 96 & 0 \\
\hline 8 & 5 & 95 & 0 & 5 & 95 & 0 & 3 & 97 & 0 & 5 & 94 & 1 \\
\hline 9 & 5 & 95 & 0 & 4 & 96 & 0 & 5 & 95 & 0 & 7 & 93 & 0 \\
\hline 10 & 5 & 95 & 0 & 3 & 97 & 0 & 4 & 96 & 0 & 5 & 95 & 0 \\
\hline \multicolumn{13}{|c|}{ Ovarian stromal cells } \\
\hline 11 & 5 & 95 & 0 & 4 & 96 & 0 & 5 & 95 & 0 & 6 & 94 & 0 \\
\hline 12 & 5 & 95 & 0 & 5 & 95 & 0 & 4 & 96 & 0 & 5 & 95 & 0 \\
\hline 13 & 5 & 95 & 0 & 5 & 95 & 0 & 7 & 93 & 0 & 6 & 94 & 0 \\
\hline 14 & 5 & 95 & 0 & 4 & 96 & 0 & 6 & 94 & 0 & 5 & 94 & 1 \\
\hline 15 & 6 & 94 & 0 & 4 & 96 & 0 & 5 & 95 & 0 & 6 & 94 & 0 \\
\hline 16 & 5 & 95 & 0 & 5 & 95 & 0 & 5 & 95 & 0 & 6 & 94 & 0 \\
\hline 17 & 5 & 95 & 0 & 4 & 96 & 0 & 4 & 96 & 0 & 7 & 93 & 0 \\
\hline 18 & 5 & 95 & 0 & 4 & 96 & 0 & 5 & 95 & 0 & 6 & 94 & 0 \\
\hline 19 & 6 & 94 & 0 & 5 & 95 & 0 & 5 & 95 & 0 & 7 & 93 & 0 \\
\hline 20 & 3 & 96 & 1 & 4 & 96 & 0 & 3 & 97 & 0 & 6 & 94 & 0 \\
\hline 21 & 5 & 95 & 0 & 5 & 95 & 0 & 5 & 95 & 0 & 5 & 95 & 0 \\
\hline 22 & 4 & 96 & 0 & 4 & 96 & 0 & 4 & 96 & 0 & 6 & 94 & 0 \\
\hline 23 & 4 & 96 & 0 & 5 & 95 & 0 & 5 & 95 & 0 & 5 & 95 & 0 \\
\hline 24 & 5 & 95 & 0 & 3 & 97 & 0 & 6 & 94 & 0 & 4 & 96 & 0 \\
\hline 25 & 4 & 96 & 0 & 5 & 95 & 0 & 3 & 97 & 0 & 5 & 95 & 0 \\
\hline 26 & 5 & 95 & 0 & 6 & 94 & 0 & 5 & 95 & 0 & 4 & 96 & 0 \\
\hline 27 & 4 & 96 & 0 & 5 & 95 & 0 & 4 & 96 & 0 & 3 & 97 & 0 \\
\hline 28 & 3 & 97 & 0 & 4 & 96 & 0 & 4 & 95 & 1 & 5 & 95 & 0 \\
\hline \multicolumn{13}{|c|}{ Myometrium } \\
\hline 29 & 5 & 95 & 0 & 3 & 97 & 0 & 3 & 97 & 0 & 3 & 97 & 0 \\
\hline 30 & 4 & 96 & 0 & 5 & 95 & 0 & 4 & 96 & 0 & 3 & 97 & 0 \\
\hline 31 & 4 & 96 & 0 & 5 & 95 & 0 & 3 & 97 & 0 & 4 & 96 & 0 \\
\hline 32 & & NA & & & NA & & & NA & & & NA & \\
\hline 33 & & NA & & & NA & & & NA & & & NA & \\
\hline 34 & 4 & 94 & 2 & 4 & 96 & 0 & 5 & 95 & 0 & 5 & 95 & 0 \\
\hline 35 & 5 & 95 & 0 & 4 & 96 & 0 & 3 & 97 & 0 & 4 & 96 & 0 \\
\hline 36 & 3 & 97 & 0 & 4 & 96 & 0 & 5 & 95 & 0 & 4 & 96 & 0 \\
\hline 37 & 4 & 95 & 1 & 3 & 97 & 0 & 5 & 95 & 0 & 5 & 95 & 0 \\
\hline 38 & 4 & 96 & 0 & 5 & 95 & 0 & 4 & 96 & 0 & 3 & 97 & 0 \\
\hline 39 & 5 & 95 & 0 & 4 & 96 & 0 & 4 & 96 & 0 & 3 & 97 & 0 \\
\hline 40 & 3 & 97 & 0 & 3 & 97 & 0 & 5 & 95 & 0 & 5 & 95 & 0 \\
\hline
\end{tabular}

$\mathrm{NA}=$ not assessable because of small number of nuclei.

of FISH procedures, interpretation of FISH signals, or tissues used. Overall, the current data are indicative of a chromosomal instability in endometriosis that could be closely related to pathogenetic events leading to endometrioid cancer. In fact, there is increasing evidence that a chromosomal instability contributes to malignant transformation and tumor progression, together with multiple single gene mutations of oncogenes and tumor suppressor genes. $^{8,9}$ Among the latter, previous studies on endometriosis focused on the role of tumor suppressor genes and found losses of PTEN, CDKN2, and p53.,.$^{5-24}$
Chromosomal aberrations occur at higher frequency in ovarian endometriosis than in extragonadal endometriosis. The increased susceptibility of ovarian endometriosis over extragonadal endometriosis to acquire genomic alterations may be due to differences in the environments to which ovarian and extragonadal endometriosis are exposed. Indeed, the ovarian stroma harbors considerably higher concentrations of mediators capable of inducing genomic changes, such as sex steroids, cytokines, and growth factors, than the peritoneal fluid, ${ }^{25}$ which may be responsible for the higher rate of chromosomal aberrations in ovarian endo- 

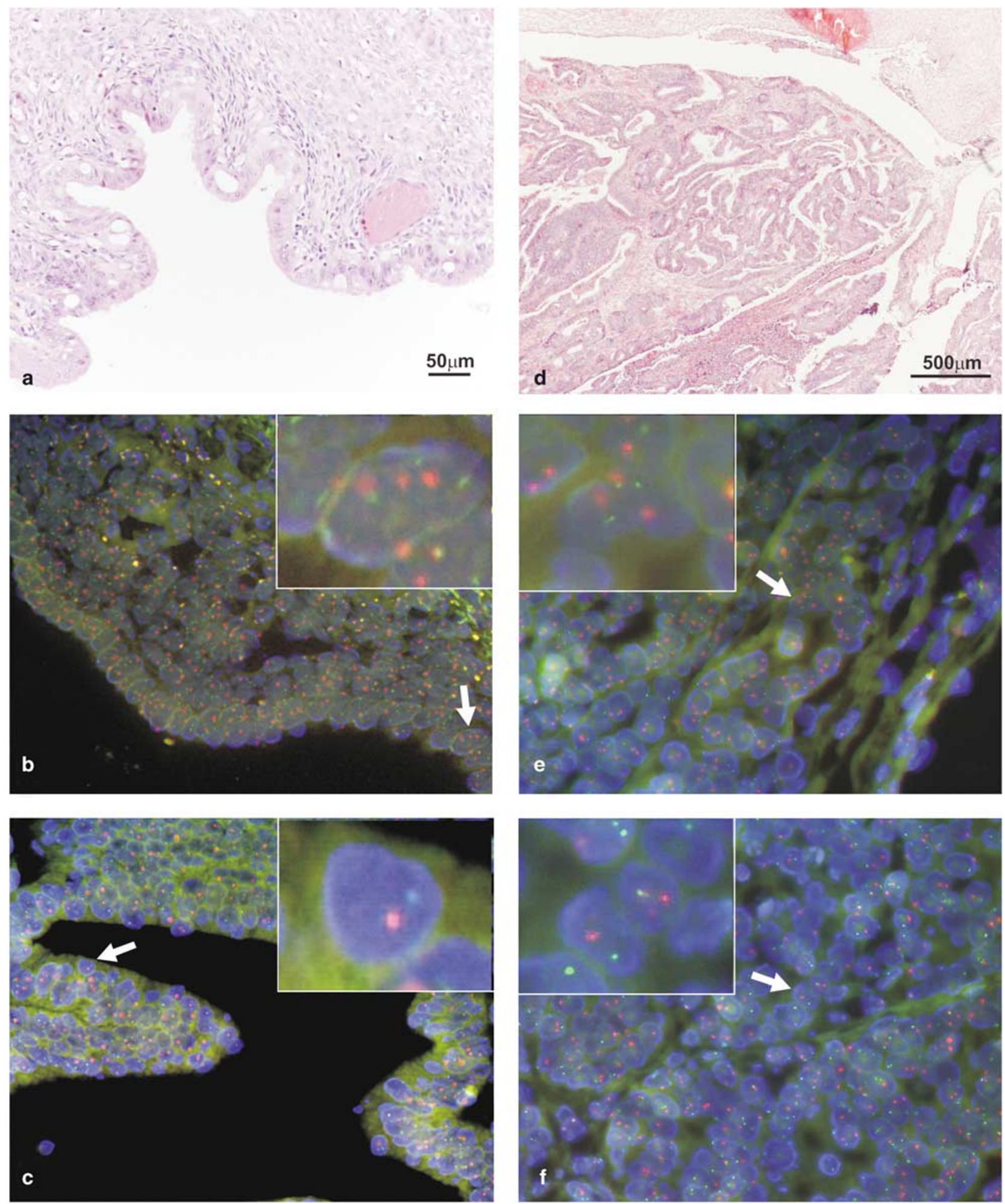

Figure 1 FISH studies showing chromosomal aberrations in endometriosis (left column) and ovarian endometrioid adenocarcinoma (right column). The H\&E-stained tissue sections show (a) an endometriotic cyst composed of glandular epithelium and adjacent endometrial stroma, and (d) an adenocarcinoma with endometrioid differentiation within an endometriotic cyst. FISH analysis shows in endometriosis in the glandular epithelium (b) trisomy of chromosome 1 (labeled green) and trisomy of chromosome 7 (labeled red), and (c) monosomy of chromosome 9 (red) and monosomy of chromosome 17 (green) (insets are higher magnification of areas indicated by arrows). Similarly, in the carcinoma, there are (e) trisomy of chromosomes 1 (green) and 7 (red) and (f) monosomy of chromosomes 9 (red) and 17 (green). 


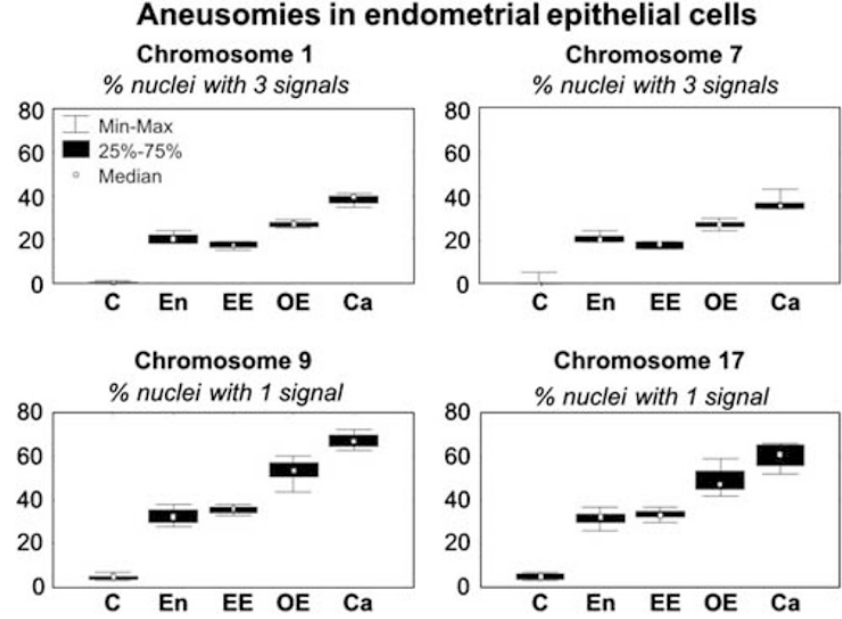

Figure 2 Semiquantitative analysis of the proportions of epithelial cells with three signals for chromosomes 1 and 7 and one signal for chromosomes 9 and 17 in control tissues (Co), normal endometrium (En; $n=12$ ), extragonadal endometriosis (EE $n=10$ ), ovarian endometriosis without carcinoma and associated with carcinoma (OE; $n=18$ ), and ovarian endometrioid adenocarcinoma (Ca; $n=8)$. The box plots indicate the percentages of aneusomic nuclei with median value (small square), distribution in quartiles (large rectangle), and total range (upper and lower bars). Significant aneusomies are present in the normal endometrium, extragonadal endometriosis, ovarian endometriosis, and ovarian endometrioid carcinomas compared with control cells. There is a significant increase in the proportions of epithelial cells with trisomies 1 and 7 , and monosomies 9 and 17 from extragonadal endometriosis to ovarian endometriosis, and from ovarian endometriosis to ovarian endometrioid carcinoma.

metriosis. Consequently, this may explain why extrauterine endometrioid adenocarcinoma associated with endometriosis develops much more frequently in the ovary than at extragonadal sites, ${ }^{26}$ despite the fact that endometriosis occurs just as frequently outside the ovary as in the ovary. ${ }^{27}$

The present data show that in endometriosis also the stromal cells harbor chromosomal aberrations. As these chromosomal aberrations are identical to those found in endometrial epithelial cells, it may be speculated that a chromosomal instability occurs in a putative common stem cell for endometrial epithelial and stromal cells. ${ }^{28}$ The presence of chromosomal instability in endometriotic stromal cells suggests that the stromal component of endometriosis may also undergo malignant transformation. This is indeed supported by the observation that extrauterine endometrial stromal tumors are frequently associated with endometriosis. ${ }^{29,30}$ The significantly lower chromosomal instability in the endometrial stromal cells compared to the epithelial cells may be related to a comparatively smaller likelihood of the endometrial stroma to progress to neoplasia and, thus, may explain the much lower incidence of extrauterine endometrioid stromal tumors with respect to extrauterine endometrioid adenocarcinoma. ${ }^{31}$

Finally, it is worthy to underscore that in the present study chromosomal aberrations were semi-

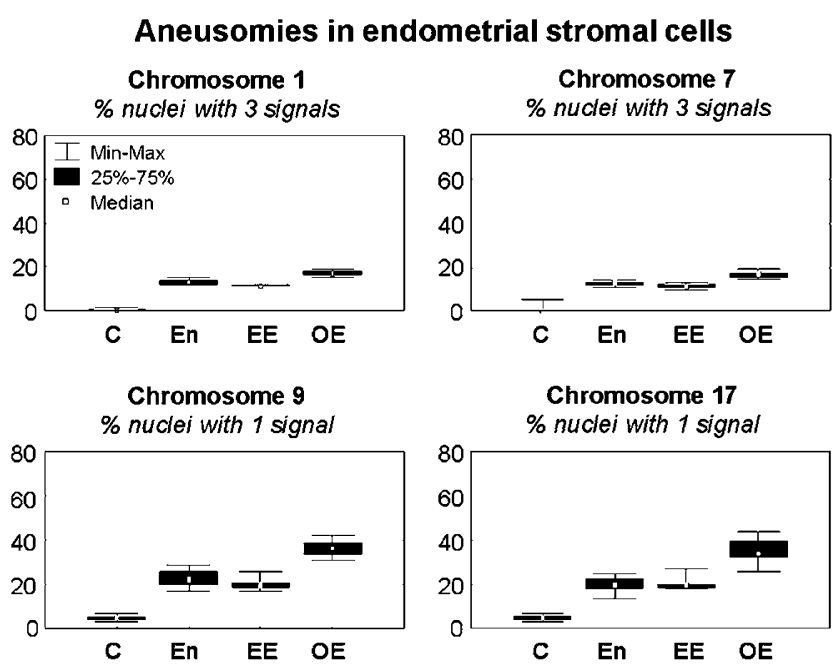

Figure 3 Semiquantitative analysis of the proportions of endometrial stromal cells with three signals for chromosomes 1 and 7 and one signal for chromosomes 9 and 17 in control tissues (Co), normal endometrium (En; $n=12$ ), extragonadal endometriosis (EE; $n=10$ ), and ovarian endometriosis (OE; $n=18)$. Endometrial stromal cells harbor small proportions of aneusomic cells. The differences in the proportions of aneusomic cells between extragonadal endometriosis and the normal endometrium are not significant, whereas ovarian endometriosis harbors significantly higher proportions of aneusomic stromal cells than extragonadal endometriosis and the normal endometrium.

quantitatively assessed as proportions of examined cells, rather than simply classified as present or absent based on cutoff values. This approach has several advantages. ${ }^{6,13}$ It allows the detection of trisomies and monosomies only occurring in small fractions of cells, which can be missed if the commonly used cutoff levels of $20 \%$ for polysomies and $40 \%$ for monosomies are applied. Furthermore, it enables analysis of small precursor lesions and of selected tissue compartments. Lastly, the comparison of results obtained in different lesions enables the study of molecular events involved in the progression of neoplastic diseases.

In conclusion, the data presented in this study demonstrate that endometriosis is characterized by several chromosomal aberrations and that it may be a precursor of gonadal and extragonadal endometrioid adenocarcinoma. We also suggest that interactions between endometriosis and the specialized ovarian stroma may be involved in the pathogenesis of ovarian cancer in general.

\section{References}

1 Heaps JM, Nieberg RK, Berek JS. Malignant neoplasms arising in endometriosis. Obstet Gynecol 1990;75: 1023-1028.

2 Feeley KM, Wells M. Precursor lesions of ovarian epithelial malignancy. Histopathology 2001;38: 87-95. 
3 Vercellini P, Parazzini F, Bolis GS, et al. Endometriosis and ovarian cancer. Am J Obstet Gynecol 1993;169: 181-182.

4 Sainz de la Cuesta R, Eichhorn JH, Rice LW, et al. Histologic transformation of benign endometriosis to early epithelial ovarian cancer. Gynecol Oncol 1996; 60:238-244.

5 Jiang X, Morland SJ, Hitchcock A, et al. Allelotyping of endometriosis with adjacent ovarian carcinoma reveals evidence of a common lineage. Cancer Res 1998;58: 1707-1712.

6 Körner M, Burckhardt E, Mazzucchelli L. Different proportions of aneusomic cells in ovarian inclusion cysts associated with serous borderline tumours and serous high-grade carcinomas support different pathogenetic pathways. J Pathol 2005;207:20-26.

7 Auersperg N, Wong AS, Choi KC, et al. Ovarian surface epithelium: biology, endocrinology, and pathology. Endocr Rev 2001;22:255-288.

8 Lengauer C, Kinzler KW, Vogelstein B. Genetic instabilities in human cancers. Nature 1998;396: 643-649.

9 Sen S. Aneuploidy and cancer. Curr Opin Oncol 2000; 12:82-88.

10 Shin JC, Ross HL, Elias S, et al. Detection of chromosomal aneuploidy in endometriosis by multicolor fluorescence in situ hybridization (FISH). Hum Genet 1997;100:401-406.

11 Kosugi Y, Elias S, Malinak LR, et al. Increased heterogeneity of chromosome 17 aneuploidy in endometriosis. Am J Obstet Gynecol 1999;180:792-797.

12 Bischoff FZ, Heard M, Simpson JL. Somatic DNA alterations in endometriosis: high frequency of chromosome 17 and p53 loss in late-stage endometriosis. J Reprod Immunol 2002;55:49-64.

13 Soldini D, Gugger M, Burckhardt E, et al. Progressive genomic alterations in intraductal papillary mucinous tumours of the pancreas and morphologically similar lesions of the pancreatic ducts. J Pathol 2003;199: 453-461.

14 Sampson JA. Endometrial carcinoma of the ovary, arising in endometrial tissue in that organ. Arch Surg 1925;10:1-27.

15 Scott RB. Malignant change in endometriosis. Obstet Gynecol 1953;2:283-289.

16 Jenkins RB, Bartelt Jr D, Stalboerger P, et al. Cytogenetic studies of epithelial ovarian carcinoma. Cancer Genet Cytogenet 1993;71:76-86.

17 Persons DL, Hartmann LC, Herath JF, et al. Interphase molecular cytogenetic analysis of epithelial ovarian carcinomas. Am J Pathol 1993;142:733-741.

18 Liehr T, Stubinger A, Thoma K, et al. Comparative interphase cytogenetics using FISH on human ovarian carcinomas. Anticancer Res 1994;14:183-188.

19 Brock JA, Liu WH, Smith ST, et al. Detection of numerical chromosome anomalies in interphase cells of ovarian carcinomas using fluorescence in situ hybridization. Genes Chromosomes Cancer 1996;16: 120-129.

20 Mazzucchelli L, Burckhardt E, Hirsiger $\mathrm{H}$, et al. Interphase cytogenetics in oncocytic adenomas and carcinomas of the thyroid gland. Hum Pathol 2000;31: 854-859.

21 Hopman AH, van Hooren E, van de Kaa CA, et al. Detection of numerical chromosome aberrations using in situ hybridization in paraffin sections of routinely processed bladder cancers. Mod Pathol 1991;4: 503-513.

22 Sato N, Tsunoda $\mathrm{H}$, Nishida $\mathrm{M}$, et al. Loss of heterozygosity on 10q23.3 and mutation of the tumor suppressor gene PTEN in benign endometrial cyst of the ovary: possible sequence progression from benign endometrial cyst to endometrioid carcinoma and clear cell carcinoma of the ovary. Cancer Res 2000;60: 7052-7056.

23 Jiang X, Hitchcock A, Bryan EJ, et al. Microsatellite analysis of endometriosis reveals loss of heterozygosity at candidate ovarian tumor suppressor gene loci. Cancer Res 1996;56:3534-3539.

24 Goumenou AG, Arvanitis DA, Matalliotakis IM, et al. Microsatellite DNA assays reveal an allelic imbalance in p16(Ink4), GALT, p53, and APOA2 loci in patients with endometriosis. Fertil Steril 2001;75: 160-165.

25 Koninckx PR, Kennedy SH, Barlow DH. Endometriotic disease: the role of peritoneal fluid. Hum Reprod Update 1998;4:741-751.

26 Clement PB. Diseases of the peritoneum. In: Kurman RJ (ed). Blaustein's Pathology of the Female Genital Tract 5th edn. Springer: New York Berlin, Heidelberg, 2002, pp 729-789.

27 Stern RC, Dash R, Bentley RC, et al. Malignancy in endometriosis: frequency and comparison of ovarian and extraovarian types. Int J Gynecol Pathol 2001;20: 133-139.

28 Gargett CE. Identification and characterisation of human endometrial stem/progenitor cells. Austr NZ J Obstet Gynaecol 2006;46:250-253.

29 Baiocchi G, Kavanagh JJ, Wharton JT. Endometrioid stromal sarcomas arising from ovarian and extraovarian endometriosis: report of two cases and review of the literature. Gynecol Oncol 1990;36:147-151.

30 Mourra N, Tiret E, Parc Y, et al. Endometrial stromal sarcoma of the rectosigmoid colon arising in extragonadal endometriosis and revealed by portal vein thrombosis. Arch Pathol Lab Med 2001;125: 1088-1090.

31 Scully RE, Young RH, Clement PB. Endometrioid tumors. In: Rosai J (ed). Atlas of Tumor Pathology: Tumors of the Ovary, Maldeveloped Gonads, Fallopian Tube, and Broad Ligament. AFIP: Washington, DC, 1998, pp 107-140. 